\title{
The horizontal branch of the Sculptor dwarf galaxy
}

\author{
Maurizio Salaris ${ }^{1}$, Thomas de Boer ${ }^{2}$, Eline Tolstoy ${ }^{3}$, Giuliana Fiorentino ${ }^{4}$, and Santi Cassisi ${ }^{5}$ \\ 1 Astrophysics Research Institute, Liverpool John Moores University, IC2, Liverpool Science Park, 146 Brownlow Hill, \\ Liverpool L3 5RF, UK \\ e-mail: M.Salaris@aljmu.ac.uk \\ 2 Institute of Astronomy, University of Cambridge, Madingley Road, Cambridge, CB3 OHA, UK \\ 3 Kapteyn Astronomical Institute, University of Groningen, Postbus 800, 9700 AV Groningen, The Netherlands \\ ${ }^{4}$ Dipartimento di Fisica e Astronomia, Università degli Studi di Bologna, Viale Berti Pichat 6/2, 40127 Bologna, Italy \\ 5 INAF - Osservatorio Astronomico di Collurania, via M. Maggini, 64100 Teramo, Italy
}

Received 19 August 2013 / Accepted 17 September 2013

\begin{abstract}
We have performed the first detailed simulation of the horizontal branch (HB) of the Sculptor dwarf spheroidal galaxy by means of synthetic modelling techniques, taking consistently into account the star formation history and metallicity evolution as determined from the main sequence and red giant branch spectroscopic observations. The only free parameter in the whole analysis is the integrated mass loss of red giant branch stars. This is the first time that synthetic HB models, consistent with the complex star formation history of a galaxy, are calculated and matched to the observed HB. We find that the metallicity range covered by the star formation history, as constrained by the red giant branch spectroscopy, plus a simple mass loss law, enable us to cover both the full magnitude and colour range of HB stars. In addition, the number count distribution along the observed $\mathrm{HB}$ can be also reproduced provided that the red giant branch mass loss is mildly metallicity dependent, with a very small dispersion at fixed metallicity. The magnitude, metallicity and period distribution of the RR Lyrae stars are also well reproduced. There is no excess of bright objects that require enhanced-He models. The lack of signatures of enhanced-He stars along the HB is consistent with the lack of the O-Na anticorrelation observed in Sculptor and other dwarf galaxies, and confirms the intrinsic difference between Local Group dwarf galaxies and globular cluster populations. We also compare the brightness of the observed red giant branch bump with the synthetic counterpart, and find a discrepancy. The theoretical bump is brighter than the observed one, which is similar to what is observed in Galactic globular clusters.
\end{abstract}

Key words. galaxies: dwarf - galaxies: evolution - galaxies: stellar content - Hertzsprung-Russell and C-M diagrams stars: horizontal-branch

\section{Introduction}

The horizontal branch $(\mathrm{HB})$ is a luminous feature seen in the colour-magnitude diagrams (CMDs) of all galaxies and globular clusters, corresponding to the central He-burning phase of low mass stars. Its shape-in a given bandpass combination-varies significantly, with an obvious dependence upon the age and metallicity range of the stellar populations of the system. However, several detailed investigations of the HB morphology in globular clusters have long since demonstrated that age and metallicity alone do not allow a unique interpretation of the HB (see, e.g., Dotter et al. 2010, and references therein).

The most critical problem is that it is not possible to predict from first principles the mass loss during the red giant branch (RGB) phase, which determines the location along the HB for a star with a fixed initial mass and chemical composition. Other factors, such as the initial helium abundance, also appear to play a crucial role in determining the form of the observed HB in Galactic globulars (see, e.g., Rood 1973; D'Antona et al. 2002; Dalessandro et al. 2011, and references therein). The role played by $\mathrm{He}$ appears to be related to the recently emerged new picture of the globular cluster origin and evolution, whereby each cluster hosts first generation (FG) stars with normal He (mass fraction roughly equal to the cosmological He abundance) and $\alpha$-enhanced metal mixtures, and second generation objects with $\mathrm{C}-\mathrm{N}, \mathrm{O}-\mathrm{Na}$ (and sometimes $\mathrm{Mg}-\mathrm{Al}$ ) anticorrelations and enhanced $\mathrm{He}$ (see, e.g. Gratton et al. 2012, for a review).

So far both of these critical parameters - RGB mass loss and initial He of individual stars - have proven hard to measure (see, e.g., Origlia et al. 2002; Villanova et al. 2009, 2012; Dalessandro et al. 2013, for some determinations on a small sample of globular clusters), and thus the HB is usually carefully avoided in the interpretation of either the age or the metallicity properties of a resolved stellar population.

This is particularly unfortunate for the study of the ancient ( $>10$ Gyr old) stellar populations in nearby galaxies. These populations are the fossil record of the early stages of galaxy evolution, which provide crucial information about the epoch of formation of cosmic structures. The standard, age diagnostics for these stars are found at faint magnitudes in a CMD, compressed into the oldest main sequence (MS) turn-off region. This region of the CMD, apart from being small (in terms of magnitude and colour extension), and thus very sensitive to photometric errors, may also contain overlapping younger populations. Also, the age-metallicity degeneracy, although much better behaved than on the RGB, may still create problems in determining a unique solution for the ages and metallicities of the ancient stars. 
Stellar evolution suggests that, at least in principle, the detailed properties of stars older than $\sim 10$ Gyr could be recovered by analysing the much more luminous and extended HB, if only its morphology could be sorted out in terms useful for understanding the relation to the star formation history and metallicity evolution of the galaxy.

The purpose of this work is to see whether it is possible to accurately model the resolved HB of the Sculptor dwarf spheroidal galaxy taking into account its past star formation history ( $\mathrm{SFH}$ - By SFH we denote the star formation rate as a function of age and metal content). Sculptor has an exceptionally detailed SFH determination - derived without considering the HB - obtained by combining CMD analysis with detailed spectroscopic metallicities along the RGB (de Boer et al. 2012). We also use the well-measured RR Lyrae properties and the optical CMD of non-variable objects as constraints to determine the best synthetic HB populations that match the observed CMD, using BaSTI stellar models by Pietrinferni et al. (2004, 2006). The only free parameter in our analysis is the total amount of mass lost along the RGB, given that age and metal abundance distributions are fixed by the SFH. To the best of our knowledge, this is the first time that a complete and detailed modelling of the $\mathrm{HB}$ of a resolved galaxy has been performed. Our investigation is therefore a first step to assess the potential of synthetic HB modelling to add further constraints on a galaxy SFH, which is a particularly important issue when studying galaxies so distant that only RGB and HB stars can be resolved.

Any mismatch between the observed and predicted magnitude distribution along the HB may be a signature of the presence of enhanced-He populations - the counterpart of second generation stars in Galactic globulars - not included in the SFH determinations. This provides an important additional piece of information regarding the comparison of photometric and chemical properties of dwarf galaxies and globular clusters. To date, comparisons of chemical abundance patterns between Galactic globulars and dwarf galaxies reveal that the latter lack the abundance anticorrelations (e.g., O-Na) typical of second generation stars in individual globular clusters (see, e.g., Geisler et al. 2007) that are associated with varying degrees of He enhancement (see, e.g., Pasquini et al. 2011). By enhanced-He populations, we mean stars born with initial He mass fractions $(Y)$ larger than the cosmological He abundance $Y=0.246$ (see Coc et al. 2013, for a recent reevaluation). The BaSTI models employ initial $Y$ values that scale with the metal mass fraction $Z$ as $\mathrm{d} Y / \mathrm{d} Z \sim 1.4$, derived by considering a cosmological $Y=0.245$ and the initial solar $Z$ and $Y$ as derived from a calibration of the standard solar model (see, e.g., Pietrinferni et al. 2004). In the metallicity regime of Sculptor, the resulting Y values are practically constant, ranging between 0.245 and 0.248 , and essentially equal to the cosmological He. It is important to notice that the determination of the $\mathrm{SFH}$ - see next section - employed the same $\mathrm{d} Y / \mathrm{d} Z$ scaling, hence approximately the cosmological He across the whole $Z$ range covered by the galaxy population.

The RGB mass loss law derived from the HB modelling - a function of initial metallicity and/or age - also provides baseline values to be compared with analogous determinations on globular clusters, and to be tested on the HB of other dwarf galaxies. In the appendix, as a byproduct of our investigation, we compare the observed difference between RGB bump and HB magnitudes with the theoretical prediction based on the galaxy SFH. We also verify whether a discrepancy exists, as found in Galactic globulars (see, e.g., Di Cecco et al. 2010).

The paper is structured as follows. Section 2 briefly described the data and the SFH used in this investigation. The next two sections present our synthetic HB analysis and results, followed by our conclusions.

\section{Data}

It is well known that the Sculptor dwarf galaxy contain a predominantly old stellar population (see, e.g., Da Costa 1984), so the contamination of populations $<10$ Gyr old along the MS and overlapping the HB is minimal. Early work on the Sculptor HB by Majewski et al. (1999) was mainly qualitative and made use of simple zero-age horizontal branch (ZAHB) fitting to assess the galaxy metallicity distribution. Tolstoy et al. (2004) and de Boer et al. (2011) later confirmed that the red and blue HBs in Sculptor exhibit differences in spatial distribution that correspond to differences in ages and metallicities within the oldest population. These previous works simply highlighted that the HB is complex and age and metallicity spreads play a role in creating the complexity.

A healthy HB extended in colour from the red to the blue indicates that a significant number of RR Lyrae variable stars must populate the instability strip, as is observed. The most complete survey to date comes from Kaluzny et al. (1995), who identified 226 RR Lyrae stars and classified their light curves. Their properties are consistent with a spread in metallicities, and $[\mathrm{Fe} / \mathrm{H}]<-1.7$. Clementini et al. (2005) confirmed this by obtaining low resolution spectroscopy of 107 variables, which showed the metallicity to peak at $[\mathrm{Fe} / \mathrm{H}] \sim-1.8$, with a range covering $-2.40<[\mathrm{Fe} / \mathrm{H}]<-0.8$.

For the accurate modelling of the resolved $\mathrm{HB}$ of the Sculptor dwarf spheroidal, we make use of a deep optical $B, V$ CMD presented in de Boer et al. (2011). In the $V-(B-V)$ CMD, the HB is the most horizontal, hence most sensitive to potential He variations. The observed $\mathrm{HB}$ extends to $(B-V) \sim 0$ in the blue. Even at these colours a change of $Y$ (at fixed $Z$ ) affects both the luminosity of the ZAHB and the luminosity of the end of the HB phase. This enables us to test the presence of enhanced-He stars along the bluest part of the galaxy HB as well.

The photometry was obtained using the CTIO 4-m MOSAIC II camera, and carefully calibrated using observations of Landolt standard fields (Landolt 2007, 1992). This resulted in an accurate photometric catalogue, covering a region ranging from the Sculptor centre out to an elliptical radius $r_{\text {ell }} \leq 1 \mathrm{deg}$. The HB of Sculptor does not have an extended blue tail in the optical, and the hottest $T_{\text {eff }}$ is lower than the observed limit $(\sim 12000 \mathrm{~K})$ for the onset of radiative levitation.

The synthetic HB calculations also make use of the detailed SFH of Sculptor determined by de Boer et al. (2012). This SFH was obtained using the same optical photometry presented in de Boer et al. (2011), combined with detailed spectroscopic metallicities along the RGB, without the observed HB stars. The distance modulus $(m-M)_{V}=19.72$ and reddening $E(B-V)=0.018$ used in the SFH determination are also employed in the HB modelling. This SFH determination uses the DSEP stellar evolution models (Dotter et al. 2008), which employ the same value as BaSTI for the cosmological He, and a very similar $\mathrm{d} Y / \mathrm{d} Z \sim 1.5$, which produces essentially the same initial $Y$ for Sculptor metallicity range. There is also good agreement between the Sculptor SFH determined with DSEP or BaSTI models, as also shown by (de Boer et al. 2012).

The SFH is provided for five annuli, extending from the centre out to a radius $r_{\mathrm{ell}}=1 \mathrm{deg}$. The region of interest for our simulation comprises the innermost two annuli, with $r_{\text {ell }} \leq 0.183 \mathrm{deg}$, that include the survey of RR Lyrae variable stars by Kaluzny et al. (1995). The adopted star formation rate 
within $r_{\mathrm{ell}} \leq 0.183$ deg is displayed in Figs. 2 and 13 of de Boer et al. (2012); it is provided in terms of solar masses per year in 1 Gyr age bins, between 5 and 14 Gyr. Broadly speaking, the star formation rate peaks in the oldest age bin, and then declines slowly with time; it reaches half the peak value between 9 and $10 \mathrm{Gyr}$ ago, and decreases down to zero between 5 and 6 Gyr ago. The low star formation rate means that the age bins between 5 and 8 Gyr have a negligible impact on the resulting synthetic HB.

For each age bin, the $\mathrm{SFH}$ is further subdivided into eight $[\mathrm{Fe} / \mathrm{H}]$ bins 0.2 dex wide, covering the range between $[\mathrm{Fe} / \mathrm{H}]=-2.5$ and -0.9 . The mean value of $[\mathrm{Fe} / \mathrm{H}]$ increases slowly with decreasing mean age of the population. A range of $[\alpha / \mathrm{Fe}]$ values $(0.2$ or 0.3 dex wide $)$ is associated to each $[\mathrm{Fe} / \mathrm{H}]$ bins (see Fig. 2 of de Boer et al. 2012). The values of $[\alpha / \mathrm{Fe}]$ decrease slowly with increasing $[\mathrm{Fe} / \mathrm{H}]$ and are generally positive, except for the more metal-rich bins, where $[\alpha / \mathrm{Fe}]$ also reaches negative values down to -0.2 dex for the higher $[\mathrm{Fe} / \mathrm{H}]$ bin.

The properties of RR Lyrae stars are taken into account using Kaluzny et al. (1995) survey in the centre of Sculptor. The survey employed many short exposure images covering a wide range in time to identify the RR Lyrae stars in the central region of Sculptor and determine the light curve profiles. Their sample can be considered complete in terms of photometric and temporal sampling of the variable objects on the HB. Intensityweighted magnitudes are provided in the $V$ band, averaged over the pulsation period of each pulsator. To prevent contamination of non-variable HB features by the presence of variable stars observed at random phase, we have identified and removed the confirmed RR Lyrae variable stars from de Boer et al. (2011) data, based on spatial position. These objects were scattered to the blue, red and within the instability strip. Due to the square field of view of the Kaluzny et al. (1995) fields, compared to the elliptical region considered here, the area covered by the SFH is $\sim 30 \%$ larger than the area for which RR Lyrae measurements are available. Therefore, we will apply a $30 \%$ reduction in the number of synthetic stars in the instability strip, in our comparisons with the observed RR Lyrae populations, to account for the different size of the area covered by Kaluzny et al. (1995) observations. This also implies that there could be a relatively low number of undetected RR Lyrae variables taken at random phase (about 70 at most) scattered along and around the HB. These however do not appreciably affect the number distributions used for our analysis. As suggested by the referee, we also performed the full analysis described in the next sections for the objects with $r_{\text {ell }} \leq 0.116 \mathrm{deg}$, e.g., within the innermost region of de Boer et al. (2012) SFH determinations. This reduces the observed number of HB objects used in the analysis by a factor $\sim 2$. In this case, Kaluzny et al. (1995) field covers the whole region, implying that the RR Lyrae sample is essentially complete and also all variables can be removed from de Boer et al. (2011) photometry. The results we obtained are completely consistent with what found for the case of $r_{\mathrm{ell}} \leq 0.183 \mathrm{deg}$, which we describe in detail in the next sections.

\section{Methods}

In this section we describe the methods employed in our theoretical modelling of Sculptor HB.

\subsection{Synthetic CMD generation}

In a complex old stellar population, the morphology of the HB depends on both the chosen RGB mass loss and the input
SFH. For this reason we have completely rewritten the BaSTI synthetic CMD generator (SYNTHETIC MAN - Pietrinferni et al. 2004) to account for both a generic SFH and an arbitrary RGB total mass loss. The code now produces a full synthetic CMD that includes also a HB of arbitrary morphology for old populations.

Briefly, the code reads the full grid of BaSTI models first, including tracks from the MS to the tip of the RGB and HB tracks, for varying $Z$ and a scaled solar metal distribution (Pietrinferni et al. 2004), and the SFH of the population - in our case the SFH by de Boer et al. (2012). For each age and $[\mathrm{Fe} / \mathrm{H}]$ bin, the synthetic CMD generator first randomly draws a stellar mass $m_{i}$, within the range 0.1 and $120 M_{\odot}$ (for consistency with de Boer et al. 2012), according to a Kroupa (2001) initial mass function. A value of the age $t_{i}$ and $[\mathrm{Fe} / \mathrm{H}]_{i}$ are then drawn randomly, according to uniform probability distributions (in agreement with the derivation of the SFH by de Boer et al. 2012), within the ranges associated with the selected age and $[\mathrm{Fe} / \mathrm{H}]$ bin. In addition, a value of $[\alpha / \mathrm{Fe}]_{i}$ is also drawn randomly (uniform probability distribution) within the range prescribed by the SFH for the selected age and $[\mathrm{Fe} / \mathrm{H}]$ bin.

If the model corresponding to $m_{i}$ and the selected metal composition has a lifetime at the first thermal pulse or at carbon ignition shorter than $t_{i}$, the star will not appear in the synthetic CMD, and a new set of $m_{i},[\mathrm{Fe} / \mathrm{H}]_{i},[\alpha / \mathrm{Fe}]_{i}$ and $t_{i}$ is drawn. For a given $[\mathrm{Fe} / \mathrm{H}]_{i}$ and $[\alpha / \mathrm{Fe}]_{i}$, we consider scaled solar models with total metallicity $[\mathrm{M} / \mathrm{H}] \sim[\mathrm{Fe} / \mathrm{H}]+\log \left(0.638 \times 10^{[\alpha / \mathrm{Fe}]}+0.362\right)$, that closely mimic models with the same $[\mathrm{M} / \mathrm{H}]$ and varying $[\alpha / \mathrm{Fe}]$ in the low mass, low metallicity regime covered by the stars currently evolving in this galaxy (Salaris et al. 1993).

If the lifetime of the model with mass $m_{i}$ is longer than $t_{i}$, and $t_{i}$ is shorter than the model age at He-ignition, interpolation in age and $Z$ amongst the BaSTI tracks will determine the CMD location of this synthetic star. If $t_{i}$ is longer than the lifetime at He-ignition, but shorter than the lifetime at the first thermal pulse, the code subtracts from $m_{i}$ a specified value of the total mass loss along the RGB $\Delta M_{\mathrm{RGB}}$, to provide a value for $m_{i, \mathrm{HB}}$, e.g., the HB mass of the synthetic star with initial mass $m_{i}$. Interpolations in mass, $Z$, and age amongst the BaSTI HB tracks provide the CMD location of the synthetic star.

Finally, the synthetic magnitudes determined with the described procedures, are perturbed by a mean Gaussian photometric error to approximate the observational error of de Boer et al. (2011) data. For the magnitude range of the HB stars, the typical $1 \sigma$ photometric uncertainty of non-variable stars is $\sim 0.007$ mag in $B$ and $V$.

The values of $m_{i}$ are added until the total mass of stars formed in each SFH bin satisfies the derived star formation rates. We have often arbitrarily multiplied the star formation rates in each bin by constant factors to have a larger population of HB stars, and minimize the Poisson noise in the simulations.

It is clear from this brief sketch of the synthetic CMD calculation that the only free parameter entering our analysis is $\Delta M_{\mathrm{RGB}}$.

\subsection{Modelling RR Lyrae stars}

Only the mean $V$ magnitudes of individual pulsators are available for the RR Lyrae stars observed by Kaluzny et al. (1995). Therefore, we had to employ theoretical determinations of the instability strip (IS) colour boundaries in our synthetic CMDs, together with the pulsational equation (Di Criscienzo et al. $2004 \mathrm{~b}$ ) that provides the fundamental period (P) of a synthetic object as a function of its mass, metallicity, bolometric 
luminosity, and effective temperature. First overtone periods $\left(\mathrm{P}_{\mathrm{FO}}\right)$ are related to the fundamental ones by the relation $P=P_{\mathrm{FO}}+0.13$ (Di Criscienzo et al. 2004b). We took the boundaries of the fundamental (F) and first overtone (FO) strips from Di Criscienzo et al. (2004b), with small adjustments, within the quoted theoretical uncertainties, to account for the empirical constraints given by the period distribution determined by Kaluzny et al. (1995), which place strong constraints on the width of the IS. As an example, if the theoretical red boundary is too red, the synthetic objects reach too long periods. We have therefore adjusted the boundaries of the $\mathrm{F}$ and $\mathrm{FO}$ regions in order to match the period range covered by the observed $\mathrm{F}$ and FO pulsators as well as possible. When using the standard IS boundaries provided by Di Criscienzo et al. (2004b) from calculations with mixing length $m l=1.5 H_{\mathrm{p}}$, the synthetic objects reached excesively long periods. This constraint forced us to consider a larger value of $m l$ in the pulsational results, that shifts the red edge of the FF region to hotter temperatures and lowers temperature of the upper boundary of the F periods. Thus, we have employed the derivatives provided by Di Criscienzo et al. (2004b) to determine the IS edges at varying $\mathrm{ml}$. However, an increased $m l$ tends to move the blue edge of the FO region to the red, making the portion of the IS populated by FO pulsators too narrow. We therefore further adjusted the $\mathrm{F}$ and $\mathrm{FO}$ boundaries, within their nominal uncertainties, at fixed $m l$ by $\pm 50-100 \mathrm{~K}$ (Marconi et al. 2003).

We compare the final $V$ magnitudes of the synthetic stars that lie within the IS to the mean $V$ magnitudes of the RR Lyrae observations of Kaluzny et al. (1995). To make this comparison we must match the observational conditions by perturbing the synthetic stars with a Gaussian photometric error, $\sigma=0.05 \mathrm{mag}$, as determined from Fig. 1 of Kaluzny et al. (1995). The photometric uncertainty on the $V$ magnitude of the observed variables is greater than the photometric uncertainty in de Boer et al. (2011), because of the shorter exposure times employed by Kaluzny et al. (1995).

\subsection{Comparing models and observations}

The bottom panel of Fig. 1 displays the observed HB, divided into three regions, delimited by rectangular boxes that we denote as red, intermediate, and blue. These boxes contain, respectively, 457, 194, and 401 stars. The blue boundary of the red box and the red boundary of the intermediate box have been placed at the approximate colours of the boundaries of the instability strips in Galactic globular cluster CMDs. The region between the red and intermediate box contains mainly RR Lyrae variables, but also a few non-variable stars displayed in the $\mathrm{CMD}^{1}$. We considered a synthetic HB model for a given $\Delta M_{\mathrm{RGB}}$ choice to be a satisfactory a match to the observations when,

1. the relative number of stars in the three boxes and within the region containing the RR Lyrae IS is reproduced within the Poisson uncertainty;

2. the observed mean $V$-magnitude of the non-variable stars in each of the three boxes is matched within $0.01 \mathrm{mag}$;

\footnotetext{
1 In our simulations, for the innermost region within $r_{\text {ell }} \leq 0.116 \mathrm{deg}$ that is fully covered by Kaluzny et al. (1995) RR Lyrae observations, we also find some stars in the CMD region between red and intermediate boxes. This strengthens the case for the small number of objects within $r_{\text {ell }} \leq 0.183$ deg lying in the same region of the CMD to be mostly non-variable stars, rather than that they are RR Lyrae objects taken at random phase and not covered by Kaluzny et al. (1995) field of view.
}

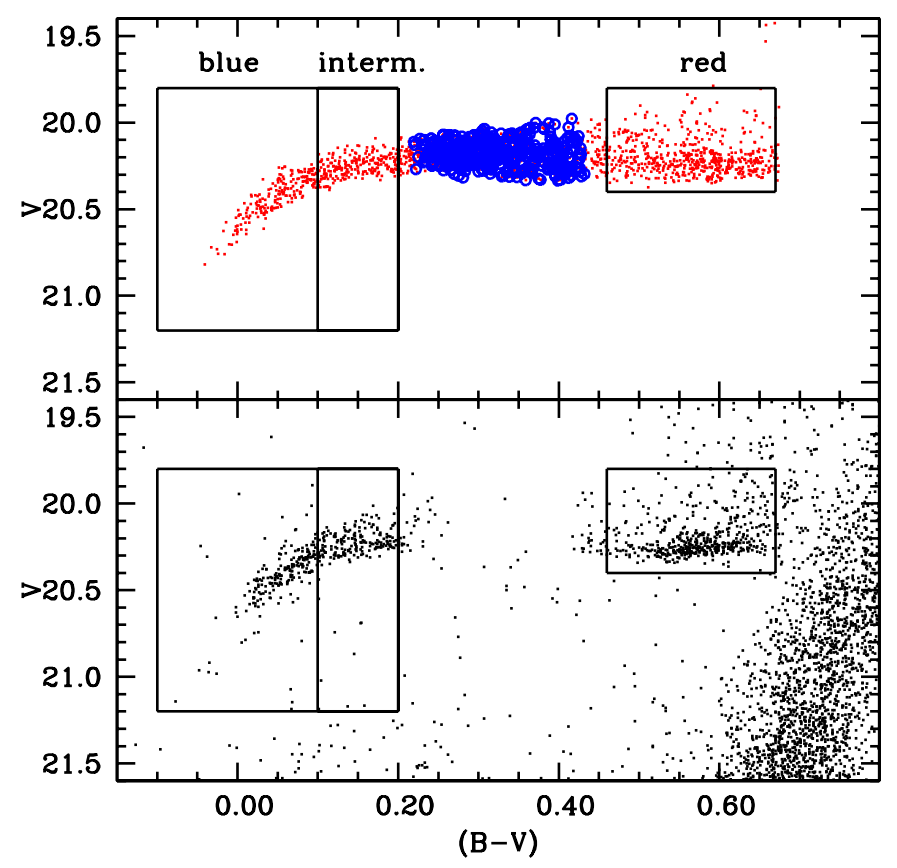

Fig. 1. Synthetic (top) vs. observed (bottom) CMD for the region in Sculptor dwarf spheoridal within $r_{\text {ell }}<0.183 \mathrm{deg}$. The synthetic CMD has a number of HB stars close to the observed number (see text for details of the simulation). The three boxes (red, intermediate, and blue) mark the three areas where star counts are compared with the simulations. The empty (blue) circles with a (red) dot represent the RR Lyrae stars found in the simulations. The observed RR Lyrae stars (as they are taken at random phase) are excluded from the bottom panel CMD. There are some non-pulsating stars in both synthetic and observed samples, located between the red and intermediate blue boxes (see text for details).

3. the total $(B-V)$ extension of the observed HB is well reproduced.

When these conditions were satisfied, we also checked that there was general agreement with the overall shapes of the histograms of observed star counts as a function of both $V$ and $(B-V)$ for the non-variable stars, and with the mean level of the RR Lyrae brightness (averaged over a pulsational cycle).

Two points must be mentioned regarding these additional constraints.

First, we could in principle have enforced the constraint of perfect statistical agreement between the theoretical and observed star counts as a function of both $V$ magnitude and colour for the non variable objects. However, a perfect fit depends on a precise knowledge of the functional dependence of $\Delta M_{\mathrm{RGB}}$ (and eventually the initial $Y$ ) on one or more stellar parameters. Given the current lack of solid theoretical and empirical guidance, this dependence may be extremely complicated, discontinuous, or involving additional physical processes not included in stellar models. We have therefore used simple parametrizations that, as we see in the next section, produce synthetic CMDs that are able to satisfy our three main criteria listed above. In this manner, we put strong constraints on $\Delta M_{\mathrm{RGB}}$ and $Y$.

Second, we determined the RR Lyrae mean magnitudes from independent observational data. The calibrated $V$ magnitudes have a maximum zero-point systematic uncertainty of $\sim 0.03 \mathrm{mag}$, according to Kaluzny et al. (1995), whilst this uncertainty is much smaller for the non-variable star photometry (de Boer et al. 2011). Given a possible small zero-point mismatch between the two photometries, we have simply 
checked that, when the main criteria were satisfied, the mean level of the synthetic RR Lyrae sample matched the observed one within the uncertainty on the relative zero-point of the two photometries. As a final, albeit weaker consistency check, we also compared the predicted and observed period distributions, and the predicted RR Lyrae $[\mathrm{Fe} / \mathrm{H}]$ distribution, with the spectroscopic observations of Clementini et al. (2005).

\section{Results}

We have calculated several synthetic CMDs, each one with typically much larger numbers of stars than observed, by appropriately scaling the SFH to minimize the Poisson error on the synthetic star counts. For each test, we fixed $\Delta M_{\mathrm{RGB}}$ and checked the agreement between synthetic and observed HB morphology. As a zero-order approximation, we tried with a standard Gaussian $\Delta M_{\mathrm{RGB}}$ and mean value (and $1 \sigma$ spread) independent of $[\mathrm{M} / \mathrm{H}]$ and age. This mass loss prescription did not match the observations for any choices of the mean value and $\sigma$ spread around the mean. Also, the use of a uniform probability with varying mean values and $\sigma$ spread, both independent of $[\mathrm{M} / \mathrm{H}]$ and age, did not help. The analysis of these failed attempts demonstrated that $\Delta M_{\mathrm{RGB}}$ must be made dependent on $[\mathrm{M} / \mathrm{H}]$, and also that only a small dispersion around these metallicity dependent mean values is consistent with the observations.

The simulations that matched the observed HB according to the criteria described in the previous section had a Gaussian distribution of $\Delta M_{\mathrm{RGB}}$ with mean values:

1. $\left\langle\Delta M_{\mathrm{RGB}}\right\rangle=0.10 M_{\odot}$ for $[\mathrm{M} / \mathrm{H}]<-1.8$;

2. $\left\langle\Delta M_{\mathrm{RGB}}\right\rangle=0.14 M_{\odot}$ for $-1.8 \leq[\mathrm{M} / \mathrm{H}] \leq-1.6$;

3. $\left\langle\Delta M_{\mathrm{RGB}}\right\rangle=0.14-0.15 M_{\odot}$ for $-1.6<[\mathrm{M} / \mathrm{H}] \leq-1.4$;

4. $\left\langle\Delta M_{\mathrm{RGB}}\right\rangle=0.14-0.16 M_{\odot}$ for $-1.4<[\mathrm{M} / \mathrm{H}] \leq-1.3$;

5. $\left\langle\Delta M_{\mathrm{RGB}}\right\rangle=0.16 M_{\odot}$ for $[\mathrm{M} / \mathrm{H}]>-1.3$;

and very small dispersions, also metallicity independent, $\sigma=$ $0.005 M_{\odot}$. We determined $\left\langle\Delta M_{\mathrm{RGB}}\right\rangle$ taking into account the error bars on the best-fit star formation rates, as provided by de Boer et al. (2012), hence the range of values for $-1.6 \leq$ $[\mathrm{M} / \mathrm{H}] \leq-1.3$. In the other metallicity ranges the uncertainty on the star formation rates causes $\left\langle\Delta M_{\mathrm{RGB}}\right\rangle$ variations below $0.01 M_{\odot}$. All discussions and figures that follow display results obtained from simulations with the best-fit value of the star formation rate. In place of step functions for $\left\langle\Delta M_{\mathrm{RGB}}\right\rangle$ vs. $[\mathrm{M} / \mathrm{H}]$ we also tried linear or quadratic analytical expressions as a function of only $[\mathrm{M} / \mathrm{H}]$ or both $[\mathrm{M} / \mathrm{H}]$ and age, but the match to the observations got generally worse.

Although the Reimers law (Reimers 1975), still widely employed to calculate RGB mass loss rates, predicts slightly increasing values of $\left\langle\Delta M_{\mathrm{RGB}}\right\rangle$ at increasing metallicity, when the free parameter $\eta$ that enters Reimers formula is kept fixed, the variations necessary to model Sculptor HB are larger. For this purpose, using the BaSTI models, we checked how our derived $\left\langle\Delta M_{\mathrm{RGB}}\right\rangle$ values can be transposed into values of the parameter $\eta$. For $[\mathrm{M} / \mathrm{H}]<-1.8\left\langle\Delta M_{\mathrm{RGB}}\right\rangle$ is slightly higher than what is predicted by $\eta=0.2$ (that provides integrated RGB mass loss values between 0.06 and $0.07 M_{\odot}$, depending on the age), whilst for $[\mathrm{M} / \mathrm{H}]>-1.8\left\langle\Delta M_{\mathrm{RGB}}\right\rangle$ is close to what is predicted by $\eta=0.4$.

With this mass loss calibration, the mean $V$ magnitudes in the red, intermediate, and blue boxes are equal to, respectively, 20.20,20.25, and 20.33, identical within 0.01 mag to the observed values. The mean brightness of the RR Lyrae IS is within 0.03 mag (the simulation being fainter) of the observed

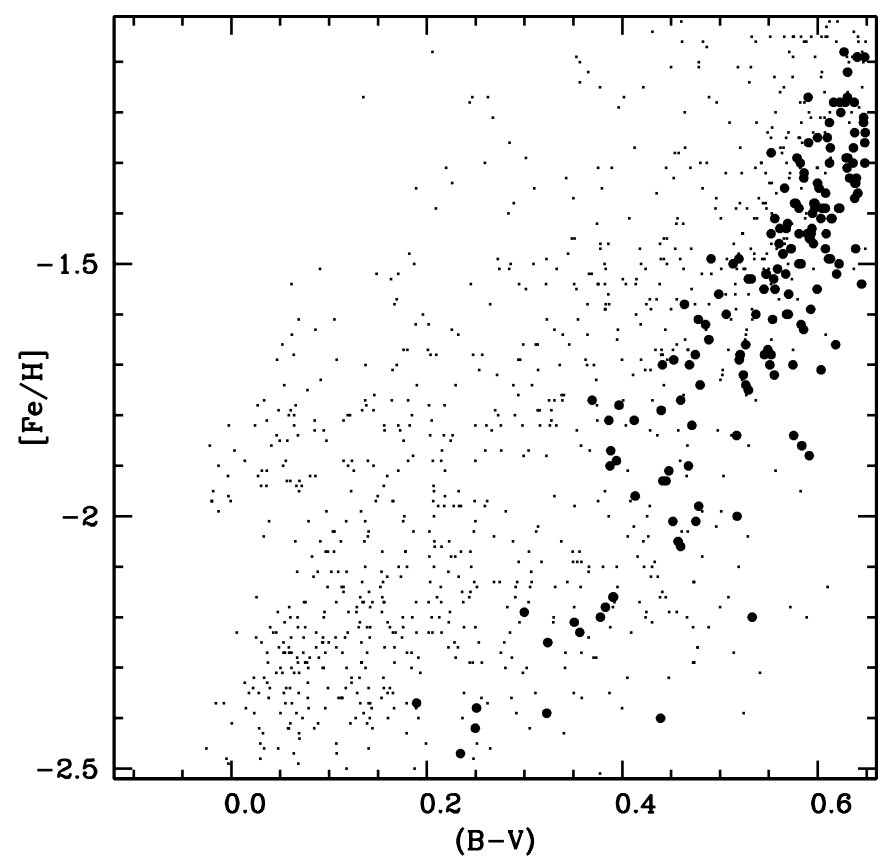

Fig. 2. $[\mathrm{Fe} / \mathrm{H}]$ as a function of $(B-V)$ for the best-fit synthetic HB sample. Dots (filled circles) denote stars with age equal or larger (smaller) than 10 Gyr. (see text for details).

value, equal to 20.14 mag. The top panel of Fig. 1 displays one realization of the $\mathrm{HB}$, without scaling the galaxy $\mathrm{SFH}$. The total absolute number of $\mathrm{HB}$ objects and the absolute number counts (not just the relative ones) in the three boxes and the region of the IS turn out to be equal, within the Poisson errors on the star counts, to the observations, considering the scaling factor for the limited area of the observed RR Lyrae population. This is an extremely important independent check of the derived star formation rates.

Figure 2 displays $[\mathrm{Fe} / \mathrm{H}]$, more directly linked to observations than $[\mathrm{M} / \mathrm{H}]$, as a function of $(B-V)$, for the HB stars in the simulation of Fig. 1. There is a general trend towards decreasing $[\mathrm{Fe} / \mathrm{H}]$ with decreasing colour, in agreement with the early analysis by Majewski et al. (1999) that made use of ZAHB fitting to the observed $\mathrm{HB}$. However, the $[\mathrm{Fe} / \mathrm{H}]$ dispersion at a given $(B-V)$ is very large. If we split the synthetic sample into objects older than $10 \mathrm{Gyr}$, and those that are younger, we obtain $[\mathrm{Fe} / \mathrm{H}]=1.46(B-V)-2.29$ for the old sample, and a much steeper dependence $[\mathrm{Fe} / \mathrm{H}]=2.80(B-V)-3.10$ for the young objects, as is also clear from Fig. 2. The dispersion of $[\mathrm{Fe} / \mathrm{H}]$ around these mean relationships is equal to $0.31 \mathrm{dex}$ for the old sample and 0.18 dex for the young one.

Figures 3 and 4 compare observed and synthetic star counts as a function of $V$ and $(B-V)$, respectively. Here we used simulations with a much larger number of objects than observed, and rescaled the star counts appropriately, to compare with observations.

The overall shape of the theoretical histograms reproduces the observed one closely. The comparison is worse for the star counts as a function of colour in the red box. It is remarkable that the magnitude distributions, very sensitive to the initial chemical composition, are well matched by the simulations, not just the mean values of $\mathrm{V}$ in the three boxes. There is no significant excess of stars brighter than what is predicted by the simulations, hence there is no clear evidence of He-rich stars. 


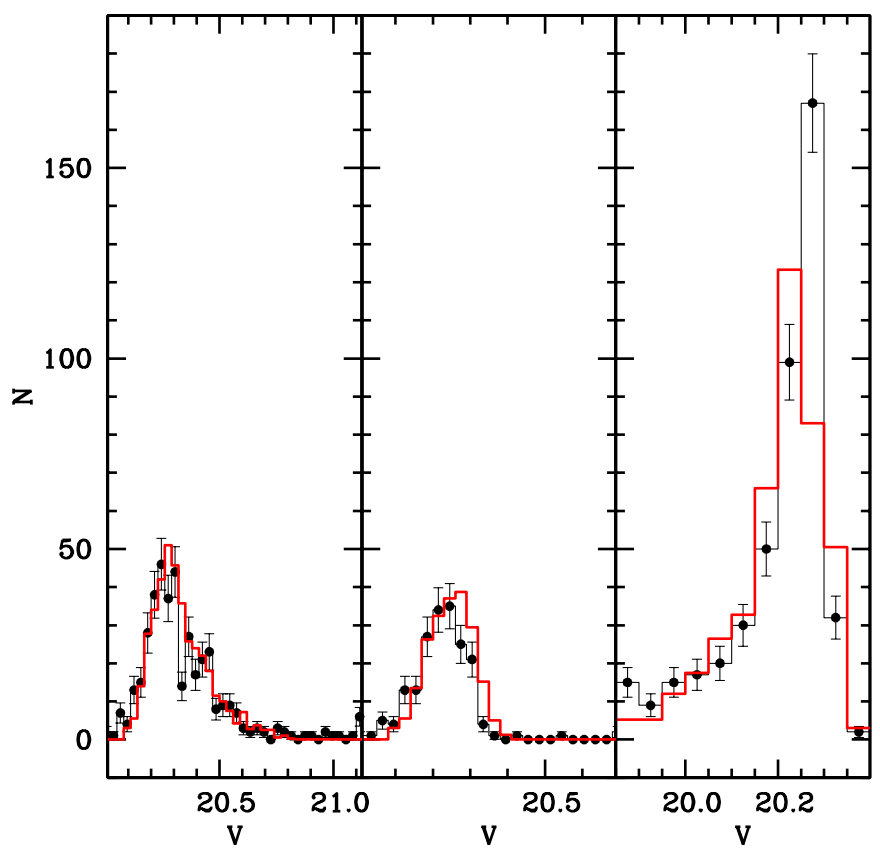

Fig. 3. Observed (solid black line and filled circles) vs. synthetic star counts (red line) as a function of the $V$ magnitude in the (from left to right) blue, intermediate, and red boxes, respectively (bin size of 0.05 mag for stars in the red box and 0.03 mag for the other two samples). Poisson errors on the observed star counts are also displayed.

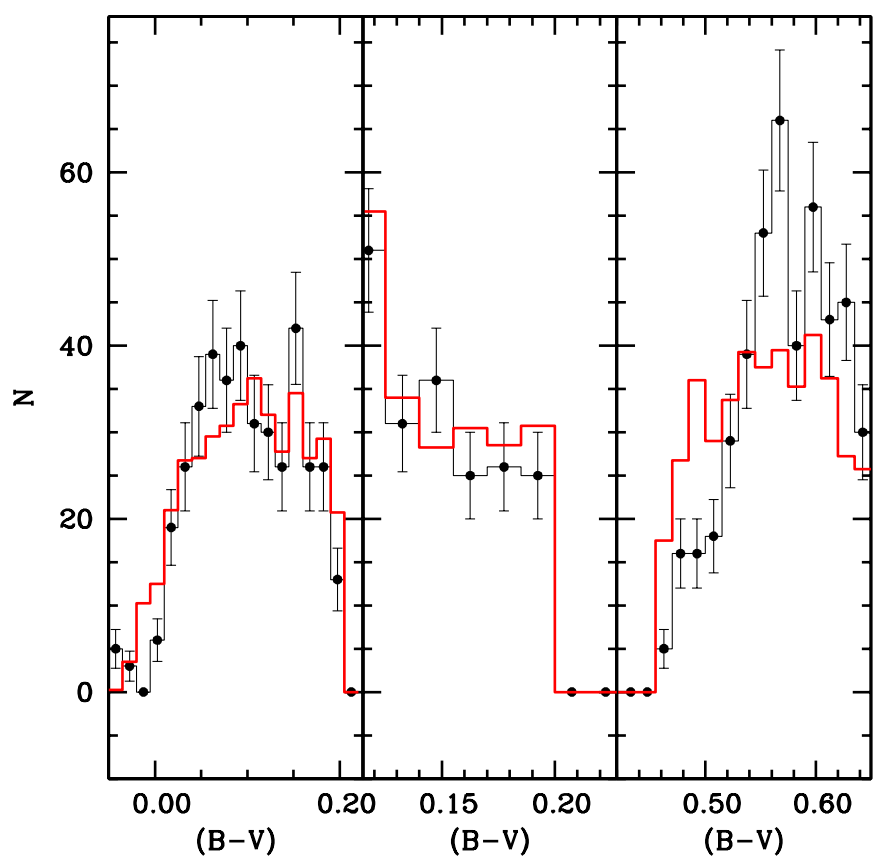

Fig. 4. As Fig. 3, except for the $(B-V)$ colour (bin size of $0.015 \mathrm{mag}$ ).

We now consider comparisons with the RR Lyrae IS, for which not only mean $V$ magnitudes, but also $[\mathrm{Fe} / \mathrm{H}]$ estimates from Clementini et al. (2005) are available, to provide an additional consistency check of the HB simulations. These authors determined $[\mathrm{Fe} / \mathrm{H}]$ for about half of the variables observed by Kaluzny et al. (1995) using a revised version of the $\Delta S$ method (Preston 1959), calibrated on both the Zinn \& West (1984) and Carretta \& Gratton (1997) (hereafter ZW and CG, respectively) globular cluster $[\mathrm{Fe} / \mathrm{H}]$ scales. Typical errors on individual $[\mathrm{Fe} / \mathrm{H}]$ estimates are equal to $\sim 0.15 \mathrm{dex}$.

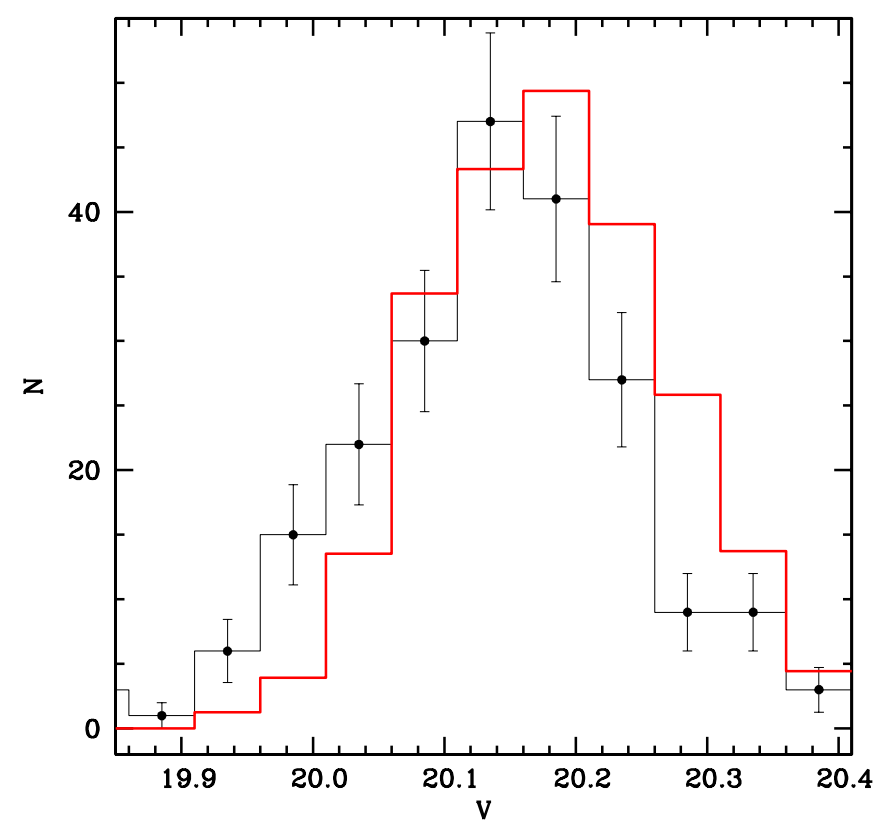

Fig. 5. As Fig. 3, except for the RR Lyrae population (bin size of $0.04 \mathrm{mag})$

Figure 5 compares observed and synthetic RR Lyrae star counts as a function of $V$. The overall shapes of the histograms are similar, with just a slight offset of the synthetic sample towards fainter magnitudes, as indicated by the mean brightness, fainter by $0.03 \mathrm{mag}$. This offset is equal to the estimated maximum zero-point error on the calibration of the observations (Kaluzny et al. 1995).

Period (data from Kaluzny et al. 1995) and [Fe/H] distributions for the sample of variables are displayed in Fig. 6. The theoretical star counts have been rescaled to account for the smaller number of objects with $[\mathrm{Fe} / \mathrm{H}]$ determinations, compared to the photometric sample of RR Lyare stars. The fit to the period distribution is not perfect, even after tuning the boundaries of the IS to match the range covered by the observations within the constraints imposed by pulsation models. Only an ad hoc tuning of the $\mathrm{F}$ and FO boundaries would provide a better match. As noticed by Di Criscienzo et al. (2004a), the IS boundaries would probably be determined better with a lower $m l$ for FO pulsators compared to F ones. This would certainly improve the fit of the $\mathrm{P}$ histogram, which displays a substantial discrepancy for the ratio of FO to F pulsators, such that it is too low in the simulation. In this respect one also has to consider that we could not simulate the so-called OR zone of the IS, where stars pulsate FO or F depending on where they evolved from (FO or F region). This adds an additional uncertainty when trying to match the observed period distribution precisely.

Regarding the metallicity distribution, we display the results for both the $\mathrm{ZW}$ and the $\mathrm{CG}[\mathrm{Fe} / \mathrm{H}]$ scales. On the whole, the synthetic sample matches the observed values on the $\mathrm{ZW}$ scale reasonably well. The average $[\mathrm{Fe} / \mathrm{H}]$ of the synthetic sample is $\langle[\mathrm{Fe} / \mathrm{H}]\rangle=-1.88$, close to the observed one on the $\mathrm{ZW}$ scale, equal to $\left\langle[\mathrm{Fe} / \mathrm{H}]_{\mathrm{ZW}}\right\rangle=-1.84$. For comparison, the observed mean value on the $\mathrm{CG}$ scale is $\left\langle[\mathrm{Fe} / \mathrm{H}]_{\mathrm{CG}}\right\rangle=-1.65$.

All these results are based on CMD simulations of the central regions of the galaxy, covered by the RR Lyrae photometry. It is impossible to completely constrain the synthetic HB models without information about the variable star population within the IS. We can, however, check whether our mass loss calibration 

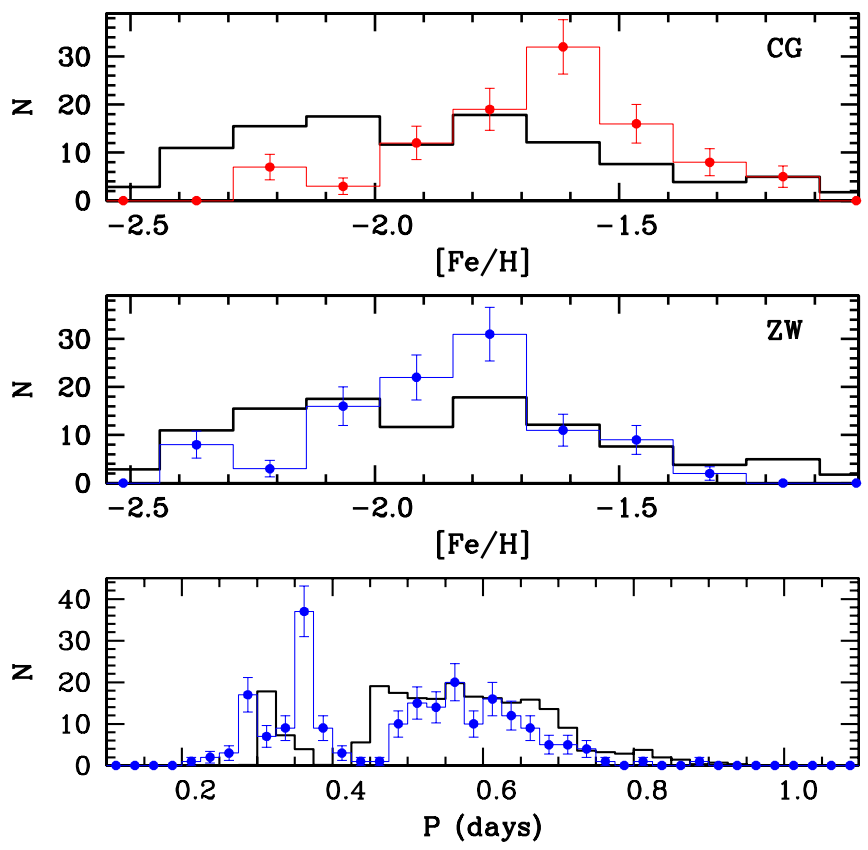

Fig. 6. Comparison with observations (displayed as histograms and filled circles with error bars) of star counts in the IS, as a function of $P$ (bin size of 0.025 days) and $[\mathrm{Fe} / \mathrm{H}]$ (bin size of $0.15 \mathrm{dex}$ ). Results for both the Zinn \& West (1984) and Carretta \& Gratton (1997) [Fe/H] scales are displayed in the middle and top panels, respectively.

based on the inner regions, is able to match the mean magnitudes and observed number of HB stars in the blue, intermediate, and red boxes of Fig. 1, when the whole observed area from the centre out to an elliptical radius $r_{\mathrm{ell}}=1 \mathrm{deg}$ is considered ${ }^{2}$. To this end, we calculated synthetic CMDs as described, considering the appropriate SFH for the whole observed area, using the same integrated RGB mass loss prescription of our best fit to the HB of the inner region. As a result, we can match within $0.01 \mathrm{mag}$ the observed mean $V$ magnitudes of stars in the three boxes, and the empirical star counts (within the associated Poisson error) in these three regions of the CMD.

\section{Conclusions}

We performed the first detailed simulation of the HB of a resolved dwarf galaxy, consistently taking into account the SFH determined from MS and RGB photometric and spectroscopic observations, and using synthetic HB techniques usually applied to study the HB in globular clusters. The number of HB stars predicted by our simulations is consistent with observations, within the Poisson error on the star counts. The colour and $V$-magnitude distribution of all non-variable HB stars in Sculptor is well matched by the synthetic model, for simple choices of the integrated RGB mass loss. The latter needs to be metallicity dependent, with a stronger dependence than predicted by the Reimers law at fixed $\eta$, to satisfy the observational constraints, and it must also have a very small dispersion at fixed metallicity. The magnitude, metallicity (on the ZW scale), and period distribution of the RR Lyrae stars are also satisfactorily reproduced, when taking the current uncertainties on the IS boundaries into account.

\footnotetext{
2 We cannot eliminate from the observed CMD the RR Lyrae stars taken at random phase, located at $r_{\text {ell }}>0.183 \mathrm{deg}$, because we cannot identify them.
}

There is no indication of enhanced-He subpopulations along the HB from the $V$-magnitude distribution of the nonvariable and variable stars, within the uncertainty on the relative photometric zero-point. The metallicity range covered by the SFH, as constrained by spectroscopy of RGB stars, plus a simple RGB mass loss law, enable us to cover both the full magnitude and colour range of HB stars. There is no excess of bright objects to be matched with enhanced-He populations. The agreement of the synthetic model with observations dictates that any enhancement of He, if present, has to be lower than $\Delta Y=0.01$. The good agreement of the synthetic sample with the $[\mathrm{Fe} / \mathrm{H}]$ distribution of the RR Lyrae stars (on the ZW scale) lend additional support to the results of our simulations.

The lack of signatures of enhanced-He stars along the HB is consistent with the lack of the $\mathrm{O}-\mathrm{Na}$ anticorrelation observed in Sculptor and other dwarf galaxies, and confirms the intrinsic difference between Local Group dwarf galaxies and globular cluster populations.

Regarding the RGB integrated mass loss $\left\langle\Delta M_{\mathrm{RGB}}\right\rangle$, our simulations suggest a simple prescription for the case of Sculptor, with $\left\langle\Delta M_{\mathrm{RGB}}\right\rangle$ slowly increasing with increasing $[\mathrm{M} / \mathrm{H}]$. We also find an extremely narrow spread around the mean values. It is natural to try and compare $\left\langle\Delta M_{\mathrm{RGB}}\right\rangle$ determined from our analysis, with similar estimates in globular clusters to assess whether, at least in case of FG stars, the RGB mass loss is approximately the same as in this galaxy. We compare our results with those of Dalessandro et al. (2011, 2013), who used synthetic HB calculations (using BaSTI models) to determine initial $Y$ and mass distribution of HB stars in a few globular clusters. For NGC 2808, a cluster with $[\mathrm{Fe} / \mathrm{H}] \sim-1.2([\mathrm{M} / \mathrm{H}] \sim-1.0$, when considering a standard value $[\alpha / \mathrm{Fe}]=0.3-0.4),\left\langle\Delta M_{\mathrm{RGB}}\right\rangle=0.15 M_{\odot}$ for FG stars, compares well with the value obtained for Sculptor at this metallicity. In the cases of M3 and M13, both with $[\mathrm{Fe} / \mathrm{H}] \sim-1.6$, the $\mathrm{HB}$ analysis provides $\left\langle\Delta M_{\mathrm{RGB}}\right\rangle=0.12 M_{\odot}$ and $0.21 M_{\odot}$, respectively. Whereas the value for M3 is reasonably close to what we derive for Sculptor at the appropriate metallicity $\left(\left\langle\Delta M_{\mathrm{RGB}}\right\rangle \sim 0.14 M_{\odot}\right)$, the result for M 13 is obviously very discrepant. On the other hand, even when $\left\langle\Delta M_{\mathrm{RGB}}\right\rangle$ values are similar to Sculptor, the dispersion around these mean values is usually larger in the globular clusters. Before drawing any strong conclusions about the similarity or differences of the integrated RGB mass loss in globular cluster FG stars and dwarf galaxies, it is clearly necessary to extend analyses like ours and Dalessandro et al. $(2011,2013)$ to much larger samples of objects.

In summary, our results show that in case of Sculptor a simple mass loss law is able to explain the observed detailed HB morphology. The next obvious step is to verify whether synthetic HB models with the same mass loss law can reproduce the $\mathrm{HB}$ of other resolved dwarf galaxies with well established and diverse SFHs. If this is the case, the combined analysis of the RGB, strongly affected by the initial metallicity, and the HB in distant galaxies, where only these phases can be detected, can provide constraints on the age and metallicity distributions of their oldest populations.

Finally, as a byproduct of our simulations, we have been able to compare the observed magnitude of the RGB bump with theory. As detailed in the Appendix, we find a discrepancy between the observed and the theoretical RGB bump, consistent with results for Galactic globulars, that point towards an excessively bright bump in stellar models, at least for intermediate metallicity and metal-poor clusters. This is at odds with recent results for Sculptor by Monelli et al. (2010); however, they based their results on older photometric data. We are able to explain the 
difference between our results for this galaxy, but the analysis of the RGB bump brightness in dwarf galaxies clearly deserves further detailed investigation.

Acknowledgements. We thank the anonymous referee for several comments that improved the presentation of our results. M.S. wishes to dedicate this paper to the memory of his father, who sadly passed away a few months ago. He also thanks the Kapteyn Astronomical Institute for their hospitality and support of a visit, during which an important point of this analysis was clarified. S.C. is grateful for financial support from PRIN-INAF 2011 "Multiple Populations in Globular Clusters: their role in the Galaxy assembly" (PI: E. Carretta), and from PRIN MIUR 2010-2011, project "The Chemical and Dynamical Evolution of the Milky Way and Local Group Galaxies”, prot. 2010LY5N2T (PI: F. Matteucci).

\section{Appendix A: The RGB bump of Sculptor}

The RGB bump is produced when the advancing H-burning shell encounters the $\mathrm{H}$-abundance discontinuity left over by the outer convection at its maximum depth reached during the first dredgeup. The resulting sudden increase in the $\mathrm{H}$-abundance in the shell alters the efficiency of the H-burning shell, and causes a temporary drop in the surface luminosity. After the shell has crossed the $\mathrm{H}$-abundance discontinuity, the luminosity starts to increase again. As a consequence, a low mass RGB star crosses the same luminosity interval three times, and a bump (a local maximum) appears in the star counts per magnitude bin (see, e.g., Iben 1968; Salaris \& Cassisi 2005).

The analysis of the photometry by Majewski et al. (1999) disclosed the presence of two distinct RGB bumps: a bright blue bump at $V \sim 19.3-19.4$, and a faint red bump one at $V \sim 20.0-20.1$. Figure A.1 displays our differential and cumulative RGB luminosity functions for Sculptor RGB and AGB stars, with $r_{\text {ell }}<0.183 \mathrm{deg}$. The break in the slope of the cumulative luminosity function, shown as dashed lines, points to the location of the RGB bump (Fusi Pecci et al. 1990). This is strongly corroborated by the CMD of Fig. A.2, that displays a tilted strip with a strong concentration of stars along the RGB, approximately centred on the magnitude of the break in the slope of the luminosity function. We thus find a single, extended, in both $V$ and $(B-V)$, continuous bump region whose ridge line is marked in Fig. A.2. The magnitude range agrees approximately with the brightness of the fainter bump claimed by Majewski et al. (1999). The bump gets fainter towards red colours as expected, given that more metal rich populations are redder along the RGB and have a fainter bump (at constant age). The spread of the bump over a wide range of magnitudes means that the feature is not well defined in the differential luminosity function.

We do not find any signature of an additional bump at $V \sim$ $19.3-19.4$, as is clear by just examining the CMD, although we find a discontinuity in the slope of the cumulative luminosity function at $V \sim 19.4$. This is approximately the magnitude of the bright bump claimed by Majewski et al. (1999), that we are going to discuss in this Appendix.

Figure A.2 also displays the ridge line of the bump region from our simulations calculated with the galaxy SFH. There is a clear offset of $\sim 0.35 \mathrm{mag}$ in $V$, where the theoretical is brighter. Notice that there is no freedom to shift the simulated CMD to fainter magnitudes because it would destroy the agreement of the synthetic HB with observations. Using the simulated $\mathrm{CMD}$ as a guide, the colour range of the observed bump corresponds to $[\mathrm{Fe} / \mathrm{H}]$ values ranging between $\sim-1.5$ and $\sim-2.0$. This disagreement is consistent with the discrepancy found in a sample of Galactic globular clusters, in approximately the same

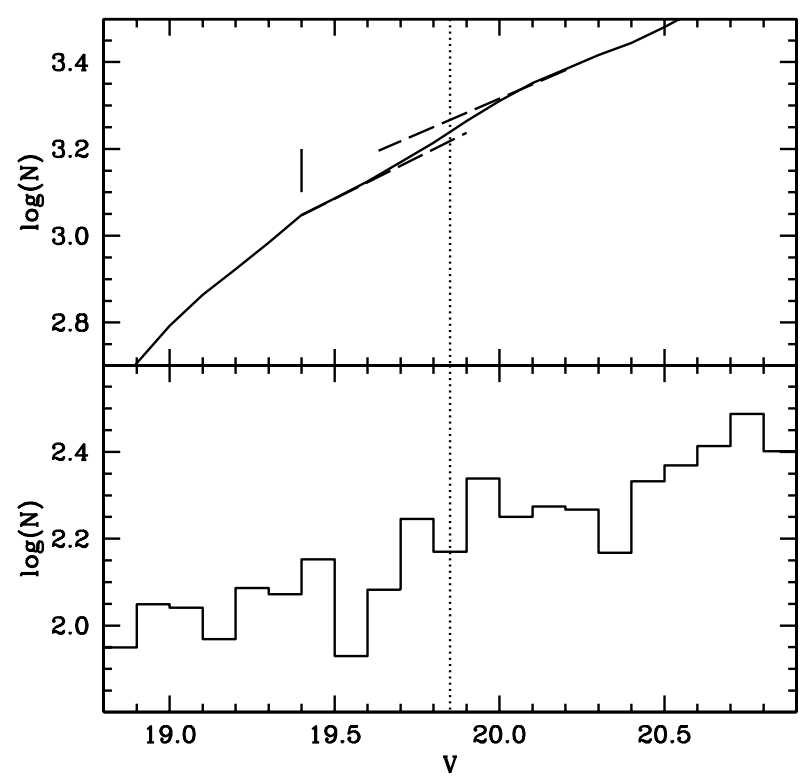

Fig. A.1. Differential (lower panel) and cumulative (upper panel) RGB luminosity functions for Sculptor RGB and AGB stars, with $r_{\text {ell }}<$ $0.183 \mathrm{deg}$. The break in the slope of the cumulative luminosity function (dashed lines) points to the location of RGB bump, whose average magnitude is marked in both panels by vertical dotted lines. The thick solid mark denotes a discontinuity in the slope of the cumulative luminosity function, that we ascribe to the appearance of the AGB clump (see text for details).

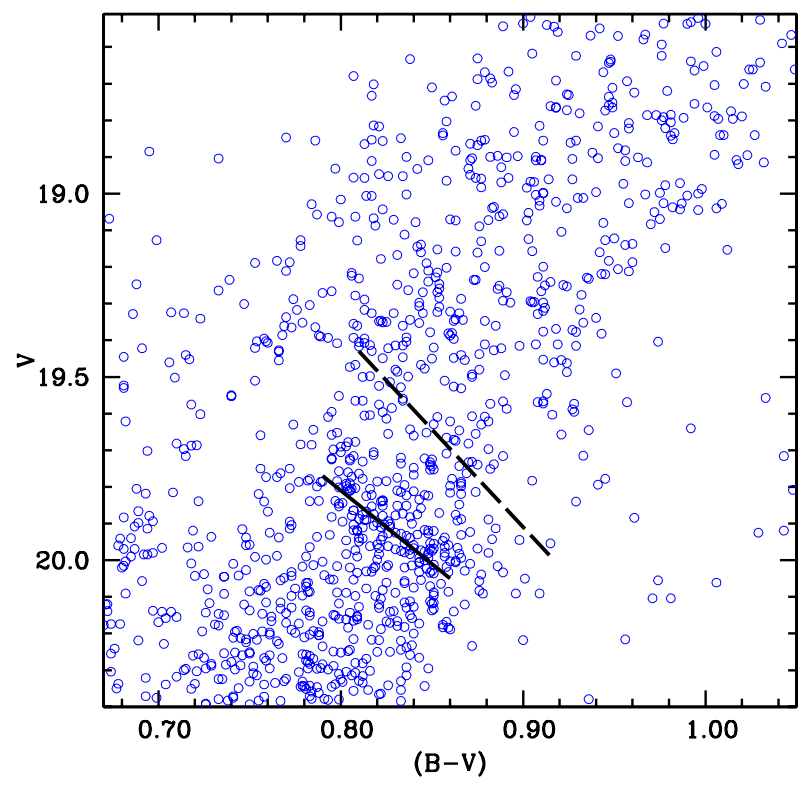

Fig. A.2. Sculptor CMD for RGB stars around the bump (for $r_{\mathrm{ell}}<$ $0.183 \mathrm{deg}$ ). The solid line marks the ridge line of the observed bump region; the dashed line denotes the ridge line of the bump for the synthetic sample of RGB stars.

metallicity interval, by Di Cecco et al. (2010) and Cassisi et al. $(2011)^{3}$.

A previous analysis by Monelli et al. (2010) compared the difference between the $V$ magnitude of the RGB bump and

3 Considering the appropriate SFH for the whole observed area within $r_{\text {ell }}=1 \mathrm{deg}$, synthetic CMDs confirm that the theoretical RGB bump is systematically brighter than the observed one, and the position of both bumps is substantially the same as for the case of $r_{\text {ell }}<0.183 \mathrm{deg}$. 


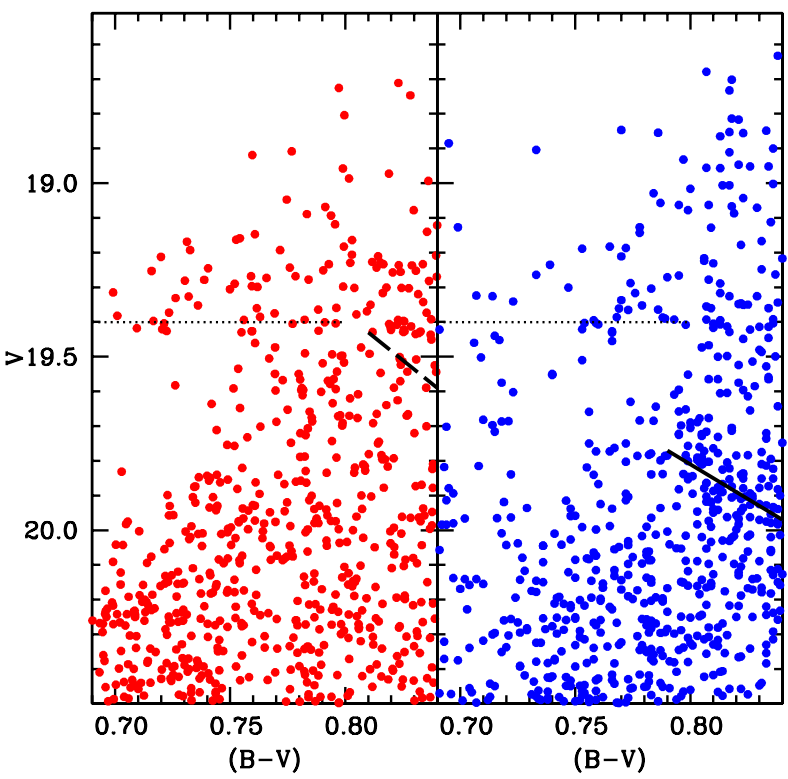

Fig. A.3. Synthetic CMD for stars around the bump and AGB clump (left panel: SFH for $r_{\mathrm{ell}}<0.183 \mathrm{deg}$ ), compared with observations (right panel). The ridge lines of both observed and synthetic RGB bumps are also marked (solid and dashed lines, respectively). The AGB clump is the feature on the blue side of the RGB, in the range $V \sim 19.1-19.4$ and $(B-V) \sim 0.70-0.75$. The dotted line marks the level of the discontinuity of the slope of the RGB+AGB cumulative luminosity function (see text for details).

the $\mathrm{HB}$ at the level of the RR Lyrae instability strip (taken at the ZAHB) $\Delta V_{\mathrm{HB}}^{\text {bump }}$ observed in Sculptor, with results from the BaSTI models. They used data from the literature that were available at that time and considered two values of $\Delta V_{\mathrm{HB}}^{\text {bump }}$, associated with the two bumps found by Majewski et al. (1999). As mentioned before, we do not find any trace of the bright bump in our adopted photometry (and there is no double RGB bump in the synthetic CMDs either), and we speculate that this may correspond to the asymptotic giant branch (AGB) clump that marks the first ignition of the He burning shell around the inert $\mathrm{CO}$ core. Essentially, AGB stars begin to contribute more substantially to the population of red giants from the level of the clump, when the evolutionary speed tends to (relatively) slow down.

Figure A.3 displays a synthetic CMD for the population with $r_{\mathrm{ell}}=0.183 \mathrm{deg}$, and approximately the same number of stars as observed. The dotted horizontal line marks the magnitude of the discontinuity of the RGB +AGB cumulative luminosity function, brighter than the RGB bump, disclosed by Fig. A.1. The AGB clump in the synthetic diagram appears in the region $(B-V) \sim$ $0.70-0.75$, on the blue side of the RGB, and $V \sim 19.1-19.4^{4}$. The dotted line essentially marks the lower envelope of AGB clump stars and agrees with the magnitude of the brighter and bluer bump claimed by Majewski et al. (1999).

We now consider the case of the fainter bump, roughly consistent with the bump region found in our data that gives $\Delta V_{\mathrm{HB}}^{\text {bump }}=-0.35 \pm 0.21$. We derived this from Majewski et al. (1999) data, by considering the ZAHB level of the red HB, that agrees with our CMD, and the faint bump, and an average $[\mathrm{M} / \mathrm{H}]-1.30 \pm 0.15$ from Kirby et al. (2009). With these

\footnotetext{
4 The clump appears slightly better defined at the position predicted by the simulations, in the observed CMD of the whole region with $r_{\mathrm{ell}}<$ $1 \mathrm{deg}$.
}

values and associated error bars, the observed $\Delta V_{\mathrm{HB}}^{\text {bump }}$ appears broadly consistent with theoretical models, for ages between 10 Gyr and 14 Gyr (see Fig. 5 of Monelli et al. 2010). Our analysis suggests instead that the theoretical $\Delta \mathrm{V}_{\mathrm{HB}}^{\text {bump }}$ is smaller than observations (the theoretical bump is too bright with respect to the HB level, taken as reference).

The difference in our result may be traced back to a combination of factors. First of all, $[\mathrm{Fe} / \mathrm{H}]$ for the stars that define the bump region lie in the range $-2.0<[\mathrm{Fe} / \mathrm{H}]<-1.5$. The $[\alpha / \mathrm{Fe}]$ values from the $\mathrm{SFH}$, combined with these $[\mathrm{Fe} / \mathrm{H}]$, give a range of $[\mathrm{M} / \mathrm{H}]$ values lower than the $[\mathrm{M} / \mathrm{H}]-1.30 \pm 0.15$ assumed by Monelli et al. (2010), and hence a brighter theoretical bump. Secondly, our HB simulations show that for the red HB (stars within the red box in our analysis) the ZAHB level, taken as a lower envelope of the observed stellar distribution, is determined by the more metal rich population, at variance with the metallicity range that dominates the bump region. Both these factors reduce the theoretical $\Delta V_{\mathrm{HB}}^{\mathrm{bump}}$ compared to the observed value, and the theoretical values in the Monelli et al. (2010) study.

\section{References}

Carretta, E., \& Gratton, R. G. 1997, A\&AS, 121, 95

Cassisi, S., Marín-Franch, A., Salaris, M., et al. 2011, A\&A, 527, A59

Clementini, G., Ripepi, V., Bragaglia, A., et al. 2005, MNRAS, 363, 734 Coc, A., Uzan, J.-P., \& Vangioni, E. 2013 [arXiv: 1307.6955]

Da Costa, G. S. 1984, ApJ, 285, 483

Dalessandro, E., Salaris, M., Ferraro, F. R., et al. 2011, MNRAS, 410, 694

Dalessandro, E., Salaris, M., Ferraro, F. R., Mucciarelli, A., \& Cassisi, S. 2013, MNRAS, 430, 459

D’Antona, F., Caloi, V., Montalbán, J., Ventura, P., \& Gratton, R. 2002, A\&A, 395,69

de Boer, T. J. L., Tolstoy, E., Saha, A., et al. 2011, A\&A, 528, A119

de Boer, T. J. L., Tolstoy, E., Hill, V., et al. 2012, A\&A, 539, A103

Di Cecco, A., Bono, G., Stetson, P. B., et al. 2010, ApJ, 712, 527

Di Criscienzo, M., Marconi, M., \& Caputo, F. 2004a, Mem. Soc. Astron. It., 75, 190

Di Criscienzo, M., Marconi, M., \& Caputo, F. 2004b, ApJ, 612, 1092

Dotter, A., Chaboyer, B., Jevremović, D., et al. 2008, ApJS, 178, 89

Dotter, A., Sarajedini, A., Anderson, J., et al. 2010, ApJ, 708, 698

Fusi Pecci, F., Ferraro, F. R., Crocker, D. A., Rood, R. T., \& Buonanno, R. 1990, A\&A, 238, 95

Geisler, D., Wallerstein, G., Smith, V. V., \& Casetti-Dinescu, D. I. 2007, PASP, 119,939

Gratton, R. G., Carretta, E., \& Bragaglia, A. 2012, A\&AR, 20, 50

Iben, Jr., I. 1968, ApJ, 154, 581

Kaluzny, J., Kubiak, M., Szymanski, M., et al. 1995, A\&AS, 112, 407

Kirby, E. N., Guhathakurta, P., Bolte, M., Sneden, C., \& Geha, M. C. 2009, ApJ, 705,328

Kroupa, P. 2001, MNRAS, 322, 231

Landolt, A. U. 1992, AJ, 104, 340

Landolt, A. U. 2007, AJ, 133, 2502

Majewski, S. R., Siegel, M. H., Patterson, R. J., \& Rood, R. T. 1999, ApJ, 520, L33

Marconi, M., Caputo, F., Di Criscienzo, M., \& Castellani, M. 2003, ApJ, 596, 299

Monelli, M., Cassisi, S., Bernard, E. J., et al. 2010, ApJ, 718, 707

Origlia, L., Ferraro, F. R., Fusi Pecci, F., \& Rood, R. T. 2002, ApJ, 571, 458

Pasquini, L., Mauas, P., Käufl, H. U., \& Cacciari, C. 2011, A\&A, 531, A35

Pietrinferni, A., Cassisi, S., Salaris, M., \& Castelli, F. 2004, ApJ, 612, 168

Pietrinferni, A., Cassisi, S., Salaris, M., \& Castelli, F. 2006, ApJ, 642, 797

Preston, G. W. 1959, ApJ, 130, 507

Reimers, D. 1975, Mem. Soc. Roy. Sci. Liège, 8, 369

Rood, R. T. 1973, ApJ, 184, 815

Salaris, M., \& Cassisi, S. 2005, Evolution of Stars and Stellar Populations (Wiley-VCH)

Salaris, M., Chieffi, A., \& Straniero, O. 1993, ApJ, 414, 580

Tolstoy, E., Irwin, M. J., Helmi, A., et al. 2004, ApJ, 617, L119

Villanova, S., Piotto, G., \& Gratton, R. G. 2009, A\&A, 499, 755

Villanova, S., Geisler, D., Piotto, G., \& Gratton, R. G. 2012, ApJ, 748, 62

Zinn, R., \& West, M. J. 1984, ApJS, 55, 45 